\title{
Loop Quantum Cosmology and Boundary Proposals
}

\author{
Martin Bojowald* \\ Max-Planck-Institute for Gravitational Physics, Albert Einstein Institute, \\ Am Mühlenberg 1, D-14476 Golm, Germany \\ Kevin Vandersloot ${ }^{\dagger}$ \\ Center for Gravitational Physics and Geometry, \\ 104 Davey Lab, University Park, PA 16802, USA
}

\begin{abstract}
For many years, the most active area of quantum cosmology has been the issue of choosing boundary conditions for the wave function of a universe. Recently, loop quantum cosmology, which is obtained from loop quantum gravity, has shed new light on this question. In this case, boundary conditions are not chosen by hand with some particular physical intuition in mind, but they are part of the dynamical law. It is then natural to ask if there are any relations between these boundary conditions and the ones provided before. After discussing the technical foundation of loop quantum cosmology which leads to crucial differences to the Wheeler-DeWitt quantization, we compare the dynamical initial conditions of loop quantum cosmology with the tunneling and the no-boundary proposal and explain why they are closer to the no-boundary condition. We end with a discussion of recent developments and several open problems of loop quantum cosmology.
\end{abstract}

\footnotetext{
*mabo@aei.mpg.de

† kfvander@gravity.psu.edu
} 


\section{INTRODUCTION}

Loop quantum cosmology is the cosmological sector of loop quantum gravity, 1.2 a canonical quantization of general relativity using Ashtekar's variables. In this formulation, gravity becomes a constrained gauge theory with the basic classical fields being an SU(2)-connection $A_{a}^{i}$, a sum of the spin connection and the extrinsic curvature, and its conjugate, the densitized triad $E_{i}^{a}$. These fields are represented in the quantum theory by holonomies of the connection and fluxes of the triad, which allows a mathematically well-defines formulation of the full theory: ${ }^{3}$ Nevertheless, the complexity of the full formalism makes it advantageous to consider simplified models in a minisuperspace quantization. Since there is a well-developed mathematical structure behind the full theory, the reduction to symmetric models can be done in such a way that essential features of the full theory, e.g., the discreteness of geometric spectra, are preserved. This is in contrast to the older Wheeler-DeWitt quantization ${ }^{4,5.6}$ where the full theory would not even be known beyond a purely formal level.

Technically, states of the full theory are functions on the space of connections which depend on $A_{a}^{i}$ only via holonomies (cylindrical functions). Symmetric states are by definition distributions on the space of connections which are supported only on the sub-manifold of invariant connections. ${ }^{7}$ It turns out that such a reduction even to the simplest cosmological models yields a quantum representation which is inequivalent to the one chosen in the Wheeler-DeWitt quantization (which is possible also in systems with a finite number of degrees of freedom since the Weyl algebra is not represented continuously) $\stackrel{8}{\text { This trans- }}$ lates into a different point of view as to the representation of basic variables. While the Wheeler-DeWitt quantization assumes that the extrinsic curvature can be promoted to a well-defined operator, the background independent techniques employed in the loop quantization allow only a representation of holonomies as operators, not the connection itself which in the isotropic setting corresponds to the extrinsic curvature. Also triad operators behave differently: they would have continuous spectra in a Wheeler-DeWitt quantization while they have discrete spectra in a loop quantization. On the other hand, these basic properties are the same in full loop quantum gravity and in loop quantum cosmology such that one can regard those cosmological models as reliable tests.

These basic technical details have far-reaching physical consequences. For instance, the Wheeler-DeWitt quantization cannot eliminate the cosmological singularities that plague 
general relativity, while they do not occur in loop quantum cosmology ${ }^{9}$ Also the issue of boundary conditions, which has been the main issue in the Wheeler-DeWitt quantization, appears in a different light. ${ }^{10,11}$ All these effects are intimately linked to the underlying discreteness of geometry.

In this contribution we describe both the basic formulation of the theory, emphasizing differences to the Wheeler-DeWitt quantization, and physical applications. Both parts can be read independently of each other.

\section{BASIC FORMALISM OF LOOP QUANTUM GRAVITY AND LOOP QUAN- TUM COSMOLOGY}

Loop quantum gravity is based on general relativity in Ashtekar's canonical variables ${ }^{12,13}$ $A_{a}^{i}=\Gamma_{a}^{i}(E)-\gamma K_{a}^{i}$ and $E_{i}^{a}$ on a spacelike slice $\Sigma$, where $\Gamma_{a}^{i}$ is the spin connection associated with the densitized triad $E_{i}^{a}, K_{a}^{i}$ the extrinsic curvature, and $\gamma$ the Barbero-Immirzi parameter which is a positive real number. For a background independent quantization it is important to represent these basic fields via holonomies

$$
h_{e}(A)=\mathcal{P} \exp \int_{e} A_{a}^{i} \tau_{i} \dot{e}^{a} d t
$$

where $e$ is a curve in $\Sigma$ with tangent vector field $\dot{e}^{a}$ and $\tau_{i}$ are Pauli matrices, and fluxes

$$
F_{S}^{(f)}(E)=\int_{S} E_{i}^{a} f^{i} n_{a} d^{2} y
$$

where $S$ is a surface in $\Sigma$ with co-normal $n_{a}$ and $f^{i}$ a test function. If all curves, surfaces and test functions are allowed, these quantities have the full information contained in the fields $A_{a}^{i}$ and $E_{i}^{a}$.

The variables are constrained by the usual SU(2)-Gauss constraint as well as the diffeomorphism constraint, which requires invariance under deformations of space, and the Hamiltonian constraint which yields the dynamics of the theory. Since the diffeomorphisms act directly on the basic fields, a representation of their classical Poisson-*-algebra has to be diffeomorphism invariant if this classical symmetry is to be preserved in the quantization. 


\section{A. Representation of the Basic Classical Algebra}

There is in fact a natural diffeomorphism invariant representation of the holonomy/flux algebra which has recently been shown to be unique. $\stackrel{14.15 .16 .17 .18}{ }$ A heuristic construction of this representation can be done as follows. Since there is a rich mathematical structure on the space of connections, it is convenient to look for the state space in the connection representation. The simplest such state is the "ground state" $\mathbf{1}(A)=1$ which does not depend on the connection at all. All higher states can then be "created" by acting with holonomies as multiplication operators. Since one can act with several holonomies, one at a time, the final states will depend on the connection non-trivially, but only along the edges in the holonomies chosen, and only via the holonomies (cylindrical functions). Labels of a given state, the edges of the holonomies used to construct it, can be arranged to a graph in $\Sigma$ which may have self-intersections and also knotting and linking information. Furthermore, since one can act with the same holonomy several times, one also needs a label representing the number of times a given edge occurs in the graph. Another way to include this information is by using irreducible $\mathrm{SU}(2)$-representations since acting once with a holonomy creates dependence of the state via the fundamental representation while acting several times then creates dependence according to the $\mathrm{SU}(2)$-coupling rules. An independent set of states can be seen to be given by the spin network basis $\frac{19}{\underline{ }}$ given by graphs whose edges are labeled by irreducible $\mathrm{SU}(2)$-representations, or half-integer spin labels $j$.

While any such state depends on the connection only via finitely many holonomies, the

full state space is obtained as a projective limit over the set of graphs. $\frac{3.20}{\text { Thus, there }}$ is no restriction on the dependence of states on connections and one obtains a faithful representation of the infinite dimensional space of connections. This state space carries a natural diffeomorphism invariant inner product, the Ashtekar-Lewandowski inner product, which defines the Hilbert space of loop quantum gravity.

Fluxes represent the conjugate of the connection and thus act as (functional) derivative operators in the connection representation. Since the states depend on the connection via holonomies, the action of flux operators can be obtained from the chain rule. A flux operator associated with a surface $S$ has a non-trivial action on a state labeled by a graph $g$ only if $S$ and $g$ intersect each other transversally. If they do, the action is given by a sum over transversal intersection points, each contribution counting the multiplicity of the edge 
encoded in its spin label $j$. Thus, flux operators count the intersection number of the surface with the graph of a state, counting also multiplicities, which is an integer. In this way, flux operators obtain a discrete spectrum which translates to discrete spectra of other geometrical operators constructed from the densitized triad, such as area or volume. ${ }^{21.22 .23}$

\section{B. Cosmological Models}

In order to follow the reduction procedure, we need to know the sub-manifold of invariant connections for an isotropic symmetry group. Any isotropic connection can be written in the form ${ }^{24}$

$$
A_{a}^{i}=c \Lambda_{I}^{i} \omega_{a}^{I}
$$

where $\omega^{I}$ are left-invariant 1-forms on $\Sigma$ for the action of the symmetry group, $\Lambda$ is an internal triad (an $S O(3)$-matrix) which is purely gauge, and $c$ is the only gauge-invariant component of the connection. Similarly, an isotropic triad has the dual form

$$
E_{i}^{a}=p \Lambda_{i}^{I} X_{I}^{a}
$$

with left-invariant vector fields $X_{I}$ dual to $\omega^{I}$.

Isotropic states are then distributions in the full state space which are supported only on isotropic connections. Thus, gauge invariant isotropic states can depend only on the component $c$, and an explicit reduction, e.g. by restricting spin network states to isotropic connections, shows that any isotropic state can be represented as

$$
|\psi\rangle=\sum_{\mu} \psi_{\mu}|\mu\rangle
$$

where the sum is over a countable subset of $\mathbb{R}$. The isotropic states

$$
\langle c \mid \mu\rangle=e^{i \mu c}
$$

form an orthonormal basis since their inner product is given by

$$
\left\langle\mu \mid \mu^{\prime}\right\rangle=\delta_{\mu, \mu^{\prime}}
$$

which implies that the Hilbert space is non-separable (all $\mu \in \mathbb{R}$ are allowed).

In this connection representation, holonomies $e^{i \mu c}$ act as multiplication operators

$$
e^{i \mu c}\left|\mu^{\prime}\right\rangle=\left|\mu+\mu^{\prime}\right\rangle
$$


and the flux $p$ as a derivative operator

$$
\hat{p}|\mu\rangle=\frac{1}{6} \gamma \ell_{\mathrm{P}}^{2} \mu|\mu\rangle .
$$

While the Hilbert space and the action of basic operators are much simpler than in the full theory, their technical features are the same: As already noted, the Hilbert space is nonseparable. Furthermore, only holonomies, not $c$ itself are promoted to well-defined operators; it is impossible to derive an operator for $c$ from the holonomy operators by differentiation since the operator family $\left(e^{i t c}\right)_{t \in \mathbb{R}}$ is not continuous in $t$ at $t=0$ :

$$
\left\langle\mu\left|e^{i t c}\right| \mu\right\rangle=\langle\mu \mid \mu+t\rangle=\delta_{t, 0} .
$$

Also as in the full theory, the flux operator $\hat{p}$ has a discrete spectrum: its eigenstates $|\mu\rangle$ are normalizable (even though a continuous range of eigenvalues is allowed).

The results (11), (21), (3) and (41) can also be used as axioms to define isotropic loop quantum cosmology, without following the reduction from the full theory. This may then be seen as a quantization alternative to and, as we will see, different from the Wheeler-DeWitt quantization (see also 25] for a similar discussion in terms of ADM variables).

In order to compare this basic framework with that of the Wheeler-DeWitt quantization, we start with the formal Hilbert spaces. In a Wheeler-DeWitt quantization one would simply employ a Schrödinger quantization of the single degree of freedom $c$ such that the Hilbert space is $L^{2}(\mathbb{R}, d c)$ and the conjugate momentum $p$ acts as a derivative operator. The loop quantization is based on a different, inequivalent Hilbert space since it is non-separable. It can be written as $L^{2}\left(\overline{\mathbb{R}}_{\text {Bohr }}, d \lambda\right)$ where $\overline{\mathbb{R}}_{\text {Bohr }}$ is the Bohr compactification of the real line $\mathbb{R}$. It is a compact Abelian group which contains $\mathbb{R} \subset \overline{\mathbb{R}}_{\text {Bohr }}$ as a dense subset. The measure $d \lambda$ of the isotropic loop Hilbert space is the Haar measure on this compact group.

Thus, the Wheeler-DeWitt quantization and the loop quantization are based on inequivalent Hilbert spaces such that they cannot be isomorphic to each other. This is possible even with finitely many degrees of freedom since the Weyl algebra $\left(e^{i t c}, e^{i s p}\right)_{t, s \in \mathbb{R}}$ is not represented continuously in the loop representation; see (5). This assumption of the Stone-von Neumann theorem, which is usually used to prove the uniqueness of the quantum mechanical representation, is thus violated. As a consequence we have the basic differences for the operators: in the Wheeler-DeWitt quantization there is an operator $\hat{c}$, which does not exist in the loop case, and the operator $\hat{p}$ of the Wheeler-DeWitt quantization has a continuous spectrum compared to the discrete one in the loop quantization. 
On the other hand, the loop quantization of the isotropic model shares basic features with full loop quantum gravity. In the full theory, the quantum configuration space is a compactification $\overline{\mathcal{A}}$ of the classical space of connections $\mathcal{A}$ which is contained in $\overline{\mathcal{A}}$ as a dense subset. This can be seen by analyzing the projective limit construction sketched above.$^{3}$ The situation in the isotropic case is completely analogous: the isotropic space of connections $\mathcal{A}_{\text {iso }} \cong \mathbb{R}$ is extended and compactified to the quantum configuration space $\overline{\mathcal{A}}_{\text {iso }} \cong \overline{\mathbb{R}}_{\text {Bohr }}$ which contains $\mathcal{A}_{\text {iso }}$ as a dense subset. Here, the same mechanism occurs just in a simpler context. As consequences we have both in the full theory and in the isotropic case a nonseparable Hilbert space, no well-defined operators for connections, and flux operators with discrete spectra. There is a difference, though, namely that the isotropic flux operator has a continuous set of eigenvalues even though its spectrum is discrete. This property comes from a degeneracy manifest in the reduction procedure: the label $\mu$ of an isotropic state $e^{i \mu c}$ does not distinguish between the parameter length of an edge and the spin when we compare it to a holonomy. There are technical subtleties resulting from this issue that can be dealt with appropriately (see [8] for a detailed discussion of all these concepts). Isotropic models, then, provide reliable tests of basic and important features of the full theory. Calculations in these models are certainly much simpler such that explicit results can be obtained. This is already shown by the volume operator which is rather complicated in the full theory while its spectrum can be obtained explicitly in the isotropic context ${ }^{26}$ Since it plays an

important role in constructing other operators such as the Hamiltonian constraint, ${ }^{27,28}$ also the dynamical equations become more treatable. We now turn to the physical applications which can be derived in this way.

\section{COSMOLOGY}

The dynamical equation of isotropic cosmology is the Friedmann equation which is the same as the Hamiltonian constraint equation. In connection variables, the isotropic gravitational part of the constraint reads

$$
H=-12 \gamma^{-2} \kappa^{-1}\left(c(c-k)+\left(1+\gamma^{2}\right) k^{2} / 4\right) \sqrt{|p|}
$$

with the gravitational constant $\kappa=8 \pi G$ and the curvature parameter $k$ which is zero for the spatially flat model and one for the closed model. In order to relate this to the usual 
form of the Friedmann equation, we need to know the relation between the basic variables $(c, p)$ and the scale factor $a$. For the triad component, one has $|p|=a^{2}$ ( $p$ can be negative, indicating the orientation of the triad), while the connection component is $c=(k-\gamma \dot{a}) / 2$. If we insert this into the constraint equation $H+H_{\text {matter }}=0$ with some matter Hamiltonian, we obtain in fact the Friedmann equation

$$
3\left(\dot{a}^{2}+k^{2}\right) a=8 \pi G a^{3} \rho(a) .
$$

\section{A. Difference Equation}

The quantization of the Hamiltonian constraint is rather complicated (see 29] for the expression) since it is a composite operator in terms of the basic ones, holonomies and fluxes. To solve the constraint, we need to act on a general state $|\psi\rangle=\sum_{\mu} \psi_{\mu}(\phi)|\mu\rangle$ where the coefficients depend on matter fields which we collectively denote as $\phi$. It is more convenient to write down the constraint equation for the coefficients $\psi_{\mu}(\phi)$ directly, i.e. to work in a triad rather than connection representation, which we can later compare to the Wheeler-DeWitt constraint. In this way, our dynamical law becomes the difference equation

$$
\begin{aligned}
& \left(V_{\mu+5}-V_{\mu+3}\right) e^{i k} \psi_{\mu+4}(\phi)-\left(2+\gamma^{2} k^{2}\right)\left(V_{\mu+1}-V_{\mu-1}\right) \psi_{\mu}(\phi) \\
& \quad+\left(V_{\mu-3}-V_{\mu-5}\right) e^{-i k} \psi_{\mu-4}(\phi)=-\frac{1}{6} \gamma^{3} \kappa \ell_{\mathrm{P}}^{2} \hat{H}_{\text {matter }}(\mu) \psi_{\mu}(\phi)
\end{aligned}
$$

using the volume eigenvalues

$$
V_{\mu}=\left(\gamma \ell_{\mathrm{P}}^{2}|\mu| / 6\right)^{3 / 2}
$$

A Wheeler-DeWitt quantization, in contrast, would result in a differential equation which turns out to be a good approximation to (17) at large volume, $|\mu| \gg 1$, where the differences can be Taylor expanded ${ }^{30}$ At small $\mu$, however, the discrete formulation is very different from the continuous one. This is crucial when we consider the fate of the classical singularity which is not removed in the Wheeler-DeWitt quantization: generically, curvatures and energy densities still diverge and the classical singularity presents a boundary to the evolution (in some special models one can argue for a more regular behavior in a different sense, see e.g. 31]). In the loop quantization, on the other hand, it turns out that there is no singularity; $; 9.32$ instead, the dynamical law provides a unique evolution from the wave function at positive $\mu$ to its values at negative $\mu$. This can be seen directly when one starts with initial data 
at large positive $\mu$ and uses (17) as a recurrence relation to evolve backward toward the classical singularity at $\mu=0$. There are no problems at first as long as the lowest order coefficient, $V_{\mu-3}-V_{\mu-5}$, in the difference equation is non-zero. For $\mu=4$, however, this coefficient is zero which means that $\psi_{0}(\phi)$ remains undetermined in this way. If this value would then appear in the next stages of the recurrence, the evolution would break down and the classical singularity would present a boundary also for the quantum evolution. As one can see, this does not occur: also for $\mu=0$ and $\mu=-4$, where $\psi_{0}$ would appear in (17) at first sight, it drops out thanks to vanishing coefficients. Here we use the fact that quantum geometry Hamiltonians imply the special feature ${ }^{33} \hat{H}_{\text {matter }}(0)=0$ which is independent of quantization ambiguities ${ }^{34}$ and comes from a general procedure in the full theory. ${ }^{28}$ Thus, $\psi_{0}$ also drops out in the matter part of the constraint equation and we can follow the evolution completely to negative $\mu$. (For a classical picture of the corresponding bounce in a closed model, see [35].)

The classical singularity, therefore, does not appear as a natural starting point where one could pose initial conditions. Nevertheless, the issue of boundary conditions appears in a new light now. ${ }^{10.11}$ When following the evolution we noticed that $\psi_{0}$ remains undetermined and we ignored the part of the constraint where it dropped out the first time $(\mu=4)$. However, this part of the constraint still has to be satisfied providing us with a linear relation between $\psi_{4}$ and $\psi_{8}$. This, in turn, translates to a linear relation between the initial data we chose. In the isotropic case this is just the right amount to specify the gravitational wave function completely up to its norm (ignoring the matter field dependence) as usually done in the Wheeler-DeWitt quantization. In order to compare with Wheeler-DeWitt wave functions we have to assume that the solution we obtain is almost constant at large volume at least for positive $\mu$, i.e. that it does not have wild oscillations at the Planck scale. This condition (pre-classicality ${ }^{10,36}$ ) ensures that the solution has a continuum limit to be compared with a Wheeler-DeWitt wave function. There are two independent such solutions which, when using the linear relation, are reduced to a unique (up to norm) linear combination. In this way, the dynamical law implies the boundary condition (dynamical initial condition) which is not chosen separately. 


\section{B. Boundary Proposals}

Boundary proposals for the Wheeler-DeWitt quantization have been studied for some time, mainly focusing on the tunneling ${ }^{37}$ and no-boundary proposals. ${ }^{38}$ We now have a very different formulation in loop quantum cosmology with a discrete evolution equation and derived boundary conditions. Nevertheless, after evolving the wave function to large volume, where the Wheeler-DeWitt quantization is a very good approximation, one can compare different boundary proposals and possible physical implications. For this purpose, we will only consider positive $\mu$ and $p$ for the rest of this section.

The first proposal is due to DeWitt who suggested that the wave function should vanish at the classical singularity, $\underset{\underline{4}}{\underline{4}} \psi(0)=0$. The dynamical initial conditions in fact coincide with this one for simple systems such as de Sitter space with flat slices (see Fig. 11) $\underline{10}^{\text {In }}$ more complicated cases, however, the discreteness at small volume implies differences in the behavior of the wave functions. In this way, the dynamical initial conditions even work for models where DeWitt's condition would not be well-posed $\underline{39}^{39}$

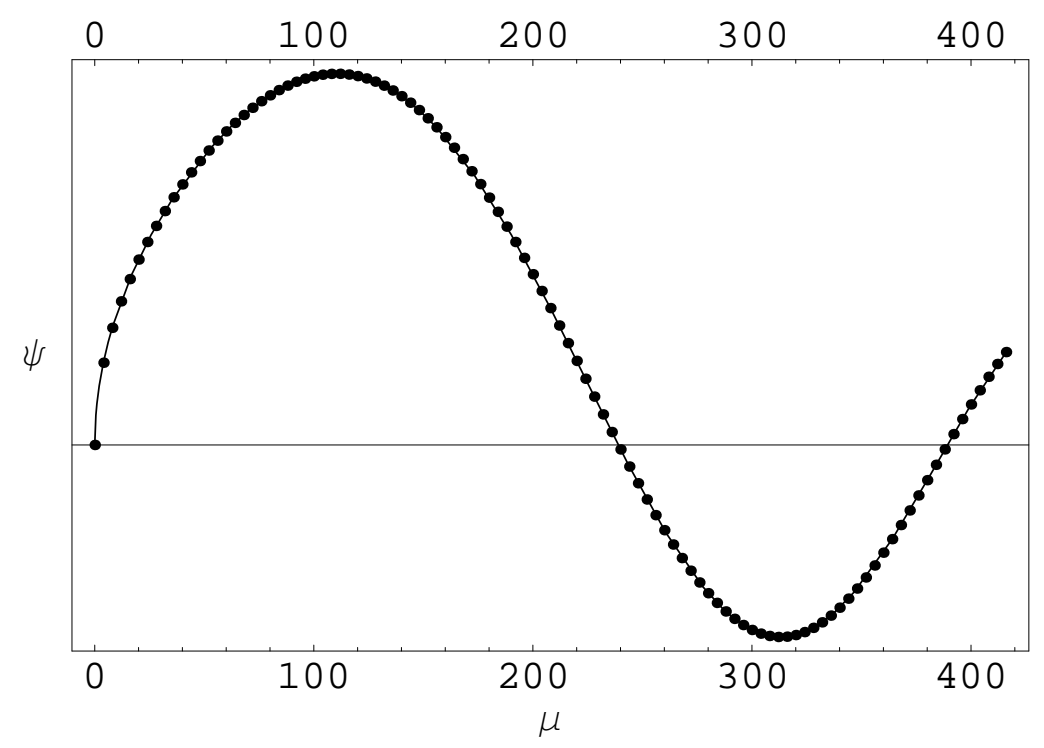

FIG. 1: Solution of the discrete equation for de Sitter space with flat slices (dots), compared to a Wheeler-DeWitt solution solving DeWitt's condition (solid line). For any other initial condition, the continuous solution would diverge at $\mu=0$ and thus deviate strongly from the discrete one.

Other boundary proposals have almost exclusively been studied for the closed model, which is in particular true for the tunneling and no-boundary proposals. In the formulation 
of the proposals, one simply considers the closed model with a cosmological constant, where there is a classically forbidden region between values of the scale factor between zero and some minimum classically allowed value. There are detailed evaluations of their different implications, but the central difference we are concerned with here is as follows. The tunneling wave function starts with a large value at $a=0$ and then decays exponentially in the classically forbidden region, which is motivated as a tunneling process of the universe. After that, the wave function becomes oscillating in the classically allowed region. The noboundary proposal, on the other hand, does have a non-vanishing exponentially increasing contribution to the wave function which will soon dominate. Thus, the wave function starts with a small value at $a=0$ and grows much higher before reaching the oscillatory phase. This different behavior is claimed to have consequences for chaotic inflation when one computes the probability distribution for the initial inflaton. This issue is still disputed, but it is often said that the no-boundary proposal prefers small initial values for the inflaton which would result in an insufficient amount of inflation.

In order to see how the loop wave function fits into this situation it is sufficient to note that the tunneling wave function is very special. It requires that the exponentially growing part does not contribute to the wave function which can be achieved only in this one very special case. Any other proposal would have a non-vanishing contribution of the growing mode and would soon differ from the tunneling wave function. Thus, any proposal which is not tailored to be exactly the tunneling one, would be much closer to the no-boundary condition at large volume. This is in particular true for the loop wave function with its dynamical initial condition. A numerical analysis (Figs. 2] and 3) shows that this wave function also increases exponentially in the classically forbidden region and thus is closer to the no-boundary proposal. Whether or not there are physically significant differences to the no-boundary wave function also at large volume remains to be studied.

This conclusion may seem bad if the no-boundary proposal does in fact not provide enough inflation. However, when we consider the evolution of a scalar or other matter field coupled to the discrete quantum geometry, there exist small-volume quantum modifications to the kinetic term of the matter Hamiltonian which automatically lead to inflation. 40 The modifications arise from the quantization of the inverse volume which removes the classical divergence In the situation of chaotic inflation where one couples an inflaton $\phi$, the modified inflaton dynamics implies that $\phi$ is driven up its potential at early stages (see Fig. 4). Before 


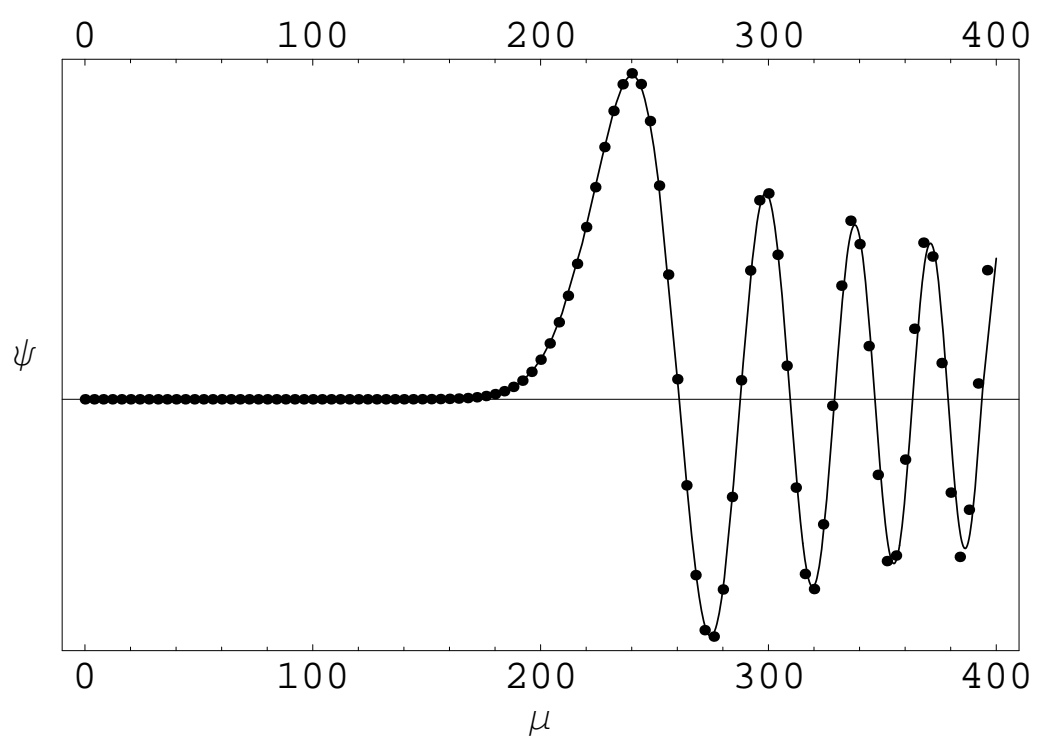

FIG. 2: Solution of the discrete equation (7) for de Sitter space with positive curvature spatial slices (dots) compared to a solution of the continuous Wheeler-DeWitt equation which is regular at $\mu=0$ (solid line).

scales much larger than the Planck length are reached, the inflaton turns around and enters a slow-roll phase of chaotic inflation. Thus, the traditional considerations of probability distributions for the inflaton are not even needed here since there is a dynamical mechanism which results in large inflaton values. This occurs for a wide range of initial conditions with the initial value of the scalar field and its conjugate momentum being constrained by the uncertainty principle. ${ }^{41}$ The particular behavior may even have observable consequences since the slow-roll condition is violated around the turning point, i.e. at early stages of the slow-roll phase translating into large current scales. ${ }^{41}$

\section{CONCLUSIONS}

The overall picture we have seen from loop quantum cosmology is very different from the traditional Wheeler-DeWitt quantization, and its structure is much richer. There are many phenomenological effects: A cut-off of classically diverging densities, ${ }^{33,34}$ which is wellstudied in flat models, implies inflation $\underline{\underline{40}}$ with possibly observable effects $\underline{41}$ when coupled to chaotic inflation. Also without coupling an inflaton, the universe would still expand with positive acceleration. Whether or not this is physically viable is undecided at this time since 


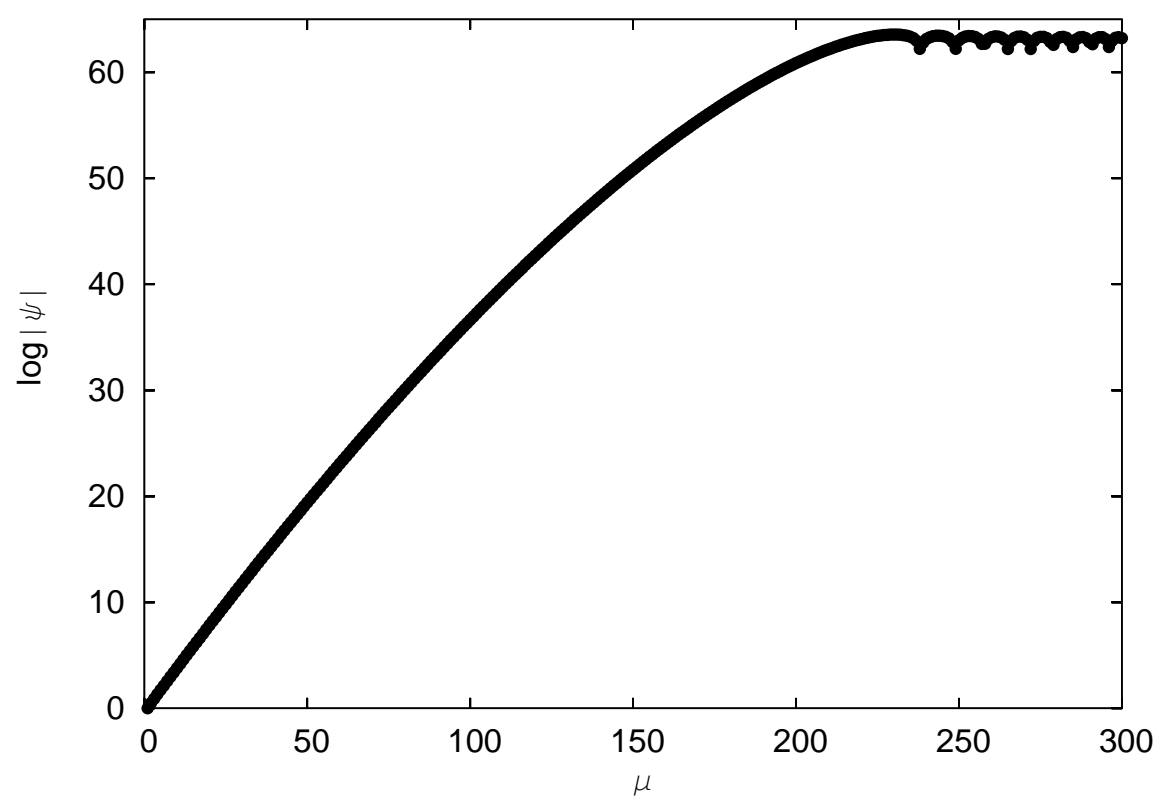

FIG. 3: Logarithm of the absolute value of the wave function. In the classically forbidden region the wave function grows by more than 60 orders of magnitude.

the usual perturbation theory becomes inapplicable. $\underline{\underline{41}}$

In the closed model with its classically forbidden region there are even more diverse phenomenological possibilities which have not yet been studied in detail. In this case, not just densities, or extrinsic curvature, experience a cut-off by quantum geometry effects, but also the intrinsic curvature can become suppressed at small volumes ${ }^{29}$ There the closed model behaves similarly to the flat model with intrinsic curvature close to zero and a classically allowed region can be created near the singularity. It remains to be seen what physical consequences these effects have as the classically allowed regions lies deep within the quantum geometry regime at small volume.

Other open issues are concerned with details of the evolution through the classical singularity. Since this involves the dynamics deep inside the Planck regime, results are very sensitive to quantization choices. This may be used to find restrictions among possible choices, but there are also many different cases which have to be studied. The equation (17) used here implies a symmetric solution which leads to equal behavior at positive or negative $\mu$. But there is a more complicated version ${ }^{32}$ which is closer to the constraint in the full theory ${ }_{27}^{27}$ The properties discussed here are insensitive to which one of those two forms for the 


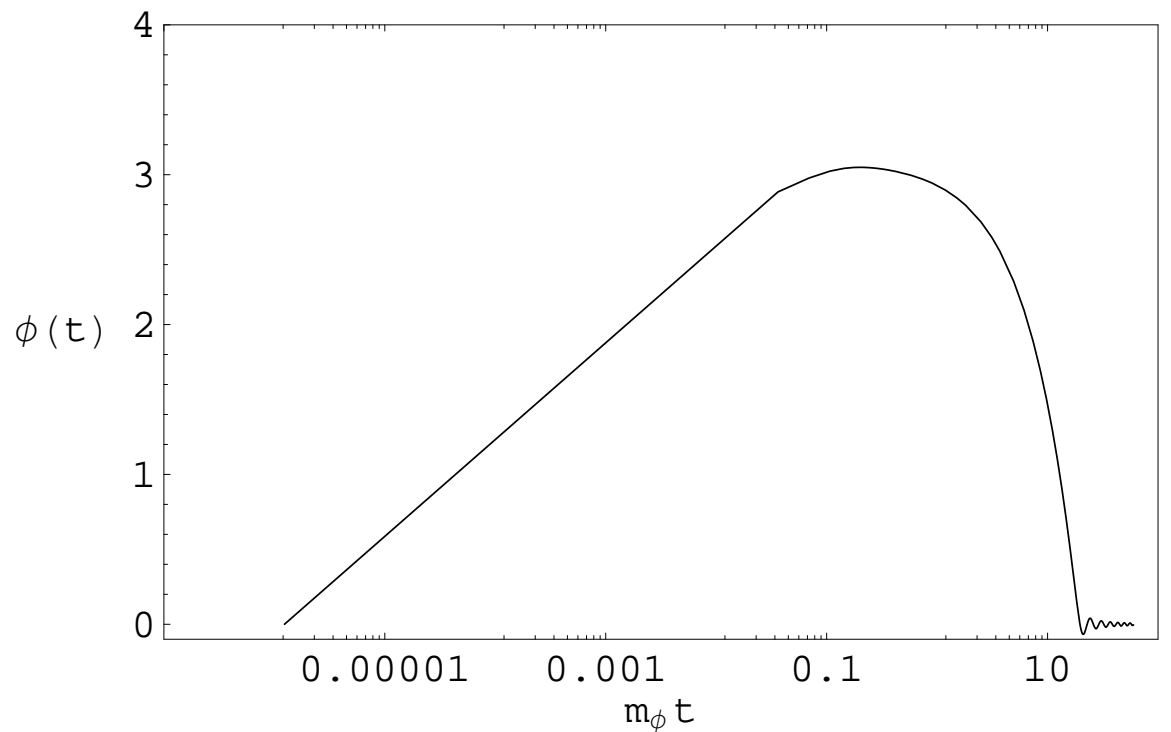

FIG. 4: Classical evolution of an inflaton field in a quadratic potential with quantum modifications to the kinetic term of the matter Hamiltonian. The quantum modifications help drive the scalar field at early stages after which slow roll inflation can occur.

constraint is used. But if we compare the wave function at positive and negative $\mu$ obtained from the more complicated constraint, then there will usually be significant small-scale oscillations at one side if they are constrained to be small at the other (see Fig. 區) $\stackrel{10}{*}$ These oscillations arise because the more complicated constraint is of higher order in internal time than the simpler one (also $\psi_{\mu \pm 8}$ are involved). Being discrete, the evolution equation may contain coefficients which do not behave smoothly especially near the classical singularity. Thus passing through the singularity a smooth wave function can have the Planck scale frequency modes excited whose amplitudes can be very large in some models. $\underline{\underline{42}}$ Particular quantizations choices can be favored if they provide an evolution equation with smooth coefficients, which may be exploited to reduce the freedom in the full quantization.

From the continuum picture point of view, the freedom for solutions to the discrete equation is reduced compared to the Wheeler-DeWitt equation. Since one has to require the existence of a continuum limit (or at least a good continuum approximation ${ }^{36}$ ) for the comparison, equation (77) is reduced to second order (even for the more complicated version mentioned in the preceding paragraph). The dynamical initial condition then leads to a reduction in the number of free parameters as seen before. From the discrete point of 


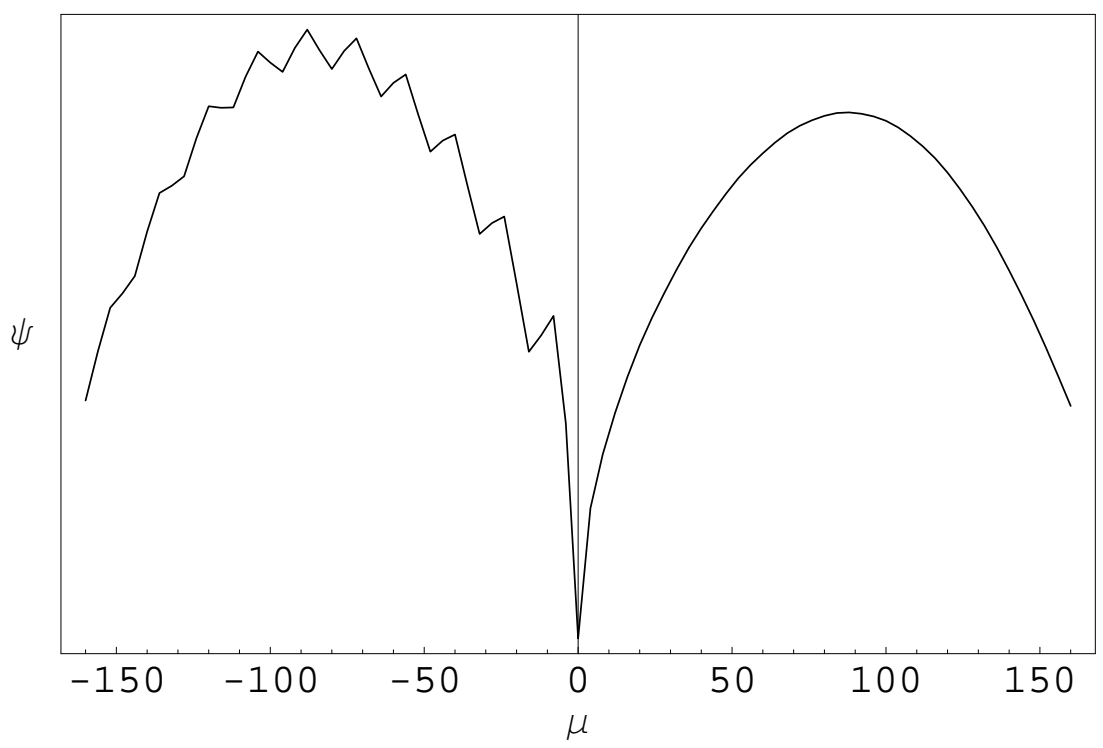

FIG. 5: Solution to the more complicated discrete equation modeled more closely to the full theory for de Sitter space with flat spatial slices. The Planck scale oscillations persist for large negative values of $\mu$. The simpler evolution equation (7) would give a wave function symmetric about $\mu=0$.

view, however, there are many more solutions (infinitely many since $\mu$ is continuous) with Planck scale oscillations. Their role and physical interpretation are open issues, and it is expected that an understanding requires the so far unknown physical inner product and the consideration of quantum observables. Tentative ideas in this direction can be found in 43. 44], and further investigations are in progress.

There are promising possibilities for future developments. It is possible to extend the results to less symmetric models, which has already been completed for anisotropic but still homogeneous ones $\stackrel{45,46}{ }$ resulting in new physical effects. $\stackrel{47}{*}$ For cosmological considerations it is also important to add inhomogeneities at least as perturbations or in midisuperspace models. This will be done in the future in order to complete the picture provided by loop quantum cosmology, but even before these technical developments much remains to be studied, as indicated above, even in isotropic models.

M. B. is grateful to C. Kiefer for an invitation to a talk at the Xth Marcel Grossmann meeting, July 20-26, 2003, Rio de Janeiro, on which this paper is based. This work was 
supported in part by NSF grant PHY00-90091 and the Eberly research funds of Penn State.

1 C. Rovelli, Loop Quantum Gravity, Living Reviews in Relativity 1, 1 (1998), gr-qc/9710008

2 T. Thiemann, Introduction to Modern Canonical Quantum General Relativity, gr-qc/0110034

3 A. Ashtekar, J. Lewandowski, D. Marolf, J. Mourão, and T. Thiemann, Quantization of Diffeomorphism Invariant Theories of Connections with Local Degrees of Freedom, J. Math. Phys. 36 (1995) 6456-6493, gr-qc/9504018

4 B. S. DeWitt, Quantum Theory of Gravity. I. The Canonical Theory, Phys. Rev. 160 (1967) $1113-1148$

5 C. W. Misner, Quantum Cosmology. I, Phys. Rev. 186 (1969) 1319-1327

6 D. L. Wiltshire, An introduction to quantum cosmology, In B. Robson, N. Visvanathan, and W. S. Woolcock, editors, Cosmology: The Physics of the Universe, pages 473-531, World Scientific, Singapore, 1996, gr-qc/0101003

7 M. Bojowald and H. A. Kastrup, Symmetry Reduction for Quantized Diffeomorphism Invariant Theories of Connections, Class. Quantum Grav. 17 (2000) 3009-3043, hep-th/9907042

8 A. Ashtekar, M. Bojowald, and J. Lewandowski, Mathematical structure of loop quantum cosmology, Adv. Theor. Math. Phys. 7 (2003) 233-268, gr-qc/0304074

9 M. Bojowald, Absence of a Singularity in Loop Quantum Cosmology, Phys. Rev. Lett. 86 (2001) 5227-5230, gr-qc/0102069

10 M. Bojowald, Dynamical Initial Conditions in Quantum Cosmology, Phys. Rev. Lett. 87 (2001) 121301, gr-qc/0104072

11 M. Bojowald, Initial Conditions for a Universe, Gen. Rel. Grav. 35 (2003) 1877-1883, gr-qc/0305069

12 A. Ashtekar, New Hamiltonian Formulation of General Relativity, Phys. Rev. D 36 (1987) $1587-1602$

13 J. F. Barbero G., Real Ashtekar Variables for Lorentzian Signature Space-Times, Phys. Rev. D 51 (1995) 5507-5510, gr-qc/9410014

14 H. Sahlmann, Some Comments on the Representation Theory of the Algebra Underlying Loop Quantum Gravity, gr-qc/0207111

15 H. Sahlmann, When Do Measures on the Space of Connections Support the Triad Operators 
of Loop Quantum Gravity?, gr-qc/0207112

16 H. Sahlmann and T. Thiemann, On the superselection theory of the Weyl algebra for diffeomorphism invariant quantum gauge theories, gr-qc/0302090

17 H. Sahlmann and T. Thiemann, Irreducibility of the Ashtekar-Isham-Lewandowski representation, gr-qc/0303074

18 A. Okolow and J. Lewandowski, Diffeomorphism covariant representations of the holonomy-flux star-algebra, gr-qc/0302059

19 C. Rovelli and L. Smolin, Spin Networks and Quantum Gravity, Phys. Rev. D 52 (1995) $5743-5759$

20 A. Ashtekar and J. Lewandowski, Projective Techniques and Functional Integration for Gauge Theories, J. Math. Phys. 36 (1995) 2170-2191

21 C. Rovelli and L. Smolin, Discreteness of Area and Volume in Quantum Gravity, Nucl. Phys. B 442 (1995) 593-619, gr-qc/9411005, Erratum: Nucl. Phys. B 456 (1995) 753

22 A. Ashtekar and J. Lewandowski, Quantum Theory of Geometry I: Area Operators, Class. Quantum Grav. 14 (1997) A55-A82, gr-qc/9602046

23 A. Ashtekar and J. Lewandowski, Quantum Theory of Geometry II: Volume Operators, Adv. Theor. Math. Phys. 1 (1997) 388-429, gr-qc/9711031

24 M. Bojowald, Loop Quantum Cosmology: I. Kinematics, Class. Quantum Grav. 17 (2000) 1489-1508, gr-qc/9910103

25 V. Husain and O. Winkler, On singularity resolution in quantum gravity, gr-qc/0312094

26 M. Bojowald, Loop Quantum Cosmology: II. Volume Operators, Class. Quantum Grav. 17 (2000) 1509-1526, gr-qc/9910104

27 T. Thiemann, Quantum Spin Dynamics (QSD), Class. Quantum Grav. 15 (1998) 839-873, gr-qc/9606089

28 T. Thiemann, QSD V: Quantum Gravity as the Natural Regulator of Matter Quantum Field Theories, Class. Quantum Grav. 15 (1998) 1281-1314, gr-qc/9705019

29 M. Bojowald and K. Vandersloot, Loop quantum cosmology, boundary proposals, and inflation, Phys. Rev. D 67 (2003) 124023, gr-qc/0303072

30 M. Bojowald, The Semiclassical Limit of Loop Quantum Cosmology, Class. Quantum Grav. 18 (2001) L109-L116, gr-qc/0105113

31 D. H. Coule, Contrasting Quantum Cosmologies, gr-qc/0312045 
M. Bojowald, Isotropic Loop Quantum Cosmology, Class. Quantum Grav. 19 (2002) 2717-2741, gr-qc/0202077

33 M. Bojowald, Inverse Scale Factor in Isotropic Quantum Geometry, Phys. Rev. D 64 (2001) 084018, gr-qc/0105067

34 M. Bojowald, Quantization ambiguities in isotropic quantum geometry, Class. Quantum Grav. 19 (2002) 5113-5130, gr-qc/0206053

35 P. Singh and A. Toporensky, Big Crunch Avoidance in k=1 Loop Quantum Cosmology, to be submitted

36 M. Bojowald and G. Date, Consistency conditions for fundamentally discrete theories, Class. Quantum Grav. 21 (2004) 121-143, gr-qc/0307083

37 A. Vilenkin, Quantum creation of universes, Phys. Rev. D 30 (1984) 509-511

38 J. B. Hartle and S. W. Hawking, Wave function of the Universe, Phys. Rev. D 28 (1983) $2960-2975$

39 M. Bojowald and F. Hinterleitner, Isotropic loop quantum cosmology with matter, Phys. Rev. D 66 (2002) 104003, gr-qc/0207038

40 M. Bojowald, Inflation from quantum geometry, Phys. Rev. Lett. 89 (2002) 261301, gr-qc/0206054

41 S. Tsujikawa, P. Singh, and R. Maartens, Loop quantum gravity effects on inflation and the CMB, astro-ph/0311015

42 F. Hinterleitner and S. Major, Isotropic Loop Quantum Cosmology with Matter II: The Lorentzian Constraint, gr-qc/0309035

43 M. Bojowald, Loop Quantum Cosmology IV: Discrete Time Evolution, Class. Quantum Grav. 18 (2001) 1071-1088, gr-qc/0008053

44 G. Mortuza Hossain, Hubble operator in isotropic loop quantum cosmology, Class. Quantum Grav. 21 (2004) 179-196, gr-qc/0308014

45 M. Bojowald, Homogeneous loop quantum cosmology, Class. Quantum Grav. 20 (2003) 25952615, gr-qc/0303073

46 M. Bojowald, G. Date, and K. Vandersloot, Homogeneous loop quantum cosmology: The role of the spin connection, gr-qc/0311004

47 M. Bojowald and G. Date, A non-chaotic quantum Bianchi IX universe and the quantum structure of classical singularities, gr-qc/0311003 